\title{
Kernos
}

Revue internationale et pluridisciplinaire de religion grecque antique

$24 \mid 2011$

Varia

\section{Archéologie cultuelle et histoire des religions antiques en Albanie}

\section{François Quantin}

\section{(2) OpenEdition}

\section{Journals}

Édition électronique

URL : http://journals.openedition.org/kernos/1967

DOI : 10.4000/kernos. 1967

ISSN : 2034-7871

Éditeur

Centre international d'étude de la religion grecque antique

Édition imprimée

Date de publication : 1 janvier 2011

Pagination : 183-204

ISSN : 0776-3824

Référence électronique

François Quantin, « Archéologie cultuelle et histoire des religions antiques en Albanie », Kernos [En ligne], 24 | 2011, mis en ligne le 01 février 2014, consulté le 20 avril 2019. URL : http:// journals.openedition.org/kernos/1967; DOI : 10.4000/kernos.1967 


\title{
Archéologie cultuelle et histoire des religions antiques en Albanie*
}

\begin{abstract}
Résumé : Dans le contexte d'une recherche en sciences humaines dont l'objectif est essentiellement, pour les dirigeants de la République populaire d'Albanie, la fondation d'une identité nationale en dehors de toute référence aux religions, quel peut être le sort d'une discipline dont l'objectif est l'identification, l'exhumation et l'analyse des vestiges de sanctuaires ? On observe une sélection des objets d'étude, et un infléchissement des résultats scientifiques. La vie religieuse antique est à la fois perçue comme un frein à l'accomplissement historique de l'Homme, et comme un naturalisme primitif proche d'une sorte de curiosité scientifique originelle. La recherche préfère restituer un folklore féérique, qui a une dimension populaire, littéraire, orale, hérö̈que, homérique. On assiste aujourd'hui à une sorte de réenchantement de ces régions, encouragé par les équipes internationales accueillies en Albanie, et fondé sur la continuité des recherches archéologiques albanaises libérées des contraintes idéologiques de l'ancien régime. Une histoire religieuse de ces régions devient possible, grâce à l'archéologie.
\end{abstract}

Abstract: For the leaders of the People's Republic of Albania, archaeology's value is limited to fostering the foundation of a national identity without any reference to religion. In such a context, what might be the fate of a field of research whose goal is the identification, analysis and exhumation of the remains of religious shrines? In this truncated discipline, subjects of investigation are carefully selected and scientific results are altered. Ancient religious life is perceived as an obstacle to the realisation of human history, although it can also be seen as a form of primitive naturalism close to primary scientific curiosity. Research prefers to participate in the restoration of a sort of magical folklore which possesses popular, literary, oral, heroic and Homeric aspects. Nowadays, a kind of "re-enchantment" of these regions seems to be occurring, and this is supported by the international archaeological teams working in Albania. This support is closely connected with a continuing (and surviving) tradition archaeological research in Albania, a tradition liberated at last from the ideological constraints of the old political regime. Therefore, a religious history of these regions becomes possible thanks to archaeology.

Avant le départ des Ottomans en 1912, l’archéologie en Albanie est étrangère. Alors qu'au sud-ouest de Ioannina la grande fouille du sanctuaire de Dodone se déroule sous la direction de Constantin Carapanos, le territoire de l'Albanie actuelle, en particulier sa partie méridionale, est parcourue par le colonel britan-

\footnotetext{
* Ce texte est dédié à la mémoire et à l'œuvre du savant archéologue que fut Skënder Anamali. Il guida en 1990 un joyeux groupe d'étudiants français emmenés par Pierre Cabanes à la découverte des sites archéologiques de son pays. Ce voyage, auquel j’ai eu la chance de participer, fut à la fois une magnifique découverte de l'Illyrie antique et une formidable initiation à l'Albanie contemporaine.
} 
nique William Martin Leake ${ }^{1}$, le clergyman Henry Holland, le révérend Christopher Wordsworth, le consul français François-Charles-Hughes-Laurent Pouqueville, ou les archéologues Léon Heuzey² et Xavier Gauthier de Claubry, membres de l'École française d'Athènes. Aucun de ces savants n'entreprit de fouilles systématiques ou régulières, et leurs contributions à l'histoire religieuse antique de ces contrées sont souvent réduites à quelques hypothèses évocatrices, à l'achat d'inscriptions et de statues, et à des réflexions sur la géographie historique de cette région du monde antique ${ }^{3}$. La seule étude quelque peu nourrie, essentiellement un relevé des vestiges et une restitution du plan du monument, est celle que consacrent Léon Heuzey et l'architecte Henri Daumet au temple de Shtyllas, dont est conservée une colonne dorique, à moins d'un kilomètre au sud d'Apollonia d'Illyrie, seule colonie grecque en place au nord d'Athènes, commentent-ils avec emphase ${ }^{4}$. Pendant la Première Guerre mondiale, des savants autrichiens mènent quelques sondages et proposent surtout des enquêtes géographiques et topographiques, nourries par de riches catalogues d'inscriptions et de sculptures qui font connaitre de nombreux documents liés à la vie religieuse antique. Dans l'entredeux-guerres, Luigi Maria Ugolini met au jour à Butrint, nous y reviendrons, un sanctuaire d'Asclépios 5 ; alors que Léon Rey à Apollonia s'intéresse à l'habitat, à la nécropole et au «centre monumental», un complexe d'édifices publics d'époques hellénistique et romaine. L'un d'eux pourrait être un modeste sanctuaire ${ }^{6}$. Néanmoins, l'Albanie de Zog s'intéresse peu aux vestiges archéologiques 7 .

L'aspect systématique et volontariste des travaux archéologiques menés après la Seconde Guerre mondiale ${ }^{8}$ pouvait laisser espérer un renouvellement profond des connaissances sur la vie religieuse en Illyrie méridionale. Elle est pourtant absente des grandes synthèses historiques, comme celle que rédigea Kristo

\footnotetext{
${ }^{1}$ Sur les motivations de W.M. Leake, $c f$. WAGSTAFF (2001).

2 Heuzey (1862), p. 7-8 : l'Empereur charge Léon Heuzey de cette mission exploratoire en Macédoine au début de 1861. Henri Daumet est ancien pensionnaire de l'Académie de Rome.

3 À la notable exception de Dodone, of. Cabanes (1996), p. 397-399, et QuAntin (2008), p. 47. Cf. CABANES (2004), p. 115-116.

${ }^{4}$ Heuzey, Daumet (1876), p. 394, et pl. 31-1 («Stylasi »).

5 Ugolini (1942).

${ }^{6}$ REY (1939); Atlas d'Apollonia, p. 212-214.

7 La création en 1922, donc avant le coup d'État d'Ahmet Zogou en décembre 1924, d'un Musée national à Tirana était l'indice d'une prise de conscience patrimoniale, of. PATSCH (1926). Fort de l'expérience acquise au Service archéologique de l'Armée d'Orient, Léon Rey crée un véritable musée archéologique à Vlora, inauguré le 8 octobre 1936, où il dépose les découvertes provenant des fouilles de la Mission française d'Apollonia (L. REY, dans P. LEMERLE, «Chronique des fouilles et découvertes archéologiques en Grèce », BCH 60 [1936], p. 474). Le musée est détruit lors du bombardement italien des 7 et 8 avril 1939 ( $c f$. P. LEMERLE, BCH 62 [1938], p. 472-473).

8 CABANES (1978) et (1986). Voir aussi le petit livre bien informé de TsônOs (2009) sur l'histoire de l'archéologie albanaise dont je dois la connaissance à Christina Mitsopoulou que je remercie vivement.
} 
Frashëri ${ }^{9}$ ou l'ouvrage que dirigea Selim Islami sur les Illyriens ${ }^{10}$, et bien entendu des manuels scolaires. Dans un manuel d'histoire pour la classe de cinquième dont le programme porte sur l'Antiquité et une partie du Moyen Âge, sur une cinquantaine de pages consacrées à la culture et à l'histoire grecques, une vingtaine de lignes seulement concernent les aspects religieux ${ }^{11}$. Le paragraphe est de plus intitulé « Besimi i grekëve, nënshtrimi i tyre ndaj fuqive të natyrës », c'està-dire «La religion des Grecs, leur soumission aux forces de la nature ». La vie religieuse romaine n'est pas abordée. La religion gréco-romaine n'est donc pas enseignée, et son évocation, de manière attendue, est réduite à une entrave à la maitrise par l'Homme de son environnement et de son destin.

Cette situation, que nous analysons dans cette étude, est remarquable car le second $\mathrm{XX}^{\mathrm{e}}$ siècle est ailleurs une époque faste pour l'histoire religieuse, qui a connu de profonds renouvellements documentaires et conceptuels. En aval, les recherches albanaises sur la vie religieuse antique sont aujourd'hui nombreuses.

Les collègues albanais qui ont accepté de répondre à mes questions à ce sujet reconnaissent que le manque d'intérêt pour l'étude des phénomènes religieux n'était pas dû à une interdiction clairement formulée, mais à une autodiscipline implicitement exigée, que certains d'entre eux n'ont pas toujours respectée. Il ne s'agit pas ici, bien entendu, d'émettre un jugement à charge ou à décharge sur l'historiographie albanaise, d'autant moins que les liens qui unissent l'archéologie et le politique sont anciens, toujours actuels et ne concernent pas seulement, bien entendu, l'Albanie et les Balkans ${ }^{12}$. Notre propos est de comprendre le faible intérêt porté par la recherche nationale à l'étude des faits religieux dans l'Antiquité, afin de mieux tirer profit de ces silences comme des travaux, qui furent néanmoins menés sur ces sujets. Raymond Aron, dans son Introduction à la philosophie de l'histoire, sous-titrée Essai sur les limites de l'objectivité bistorique, écrivait en 1948 qu'« il n'existe pas une réalité historique, toute faite avant la science, qu'il conviendrait simplement de reproduire avec fidélité. La réalité historique, parce qu'elle est humaine, est équivoque et inépuisable ${ }^{13}$. Sans prétendre l'épuiser, abordons ici quelques aspects de l'équivoque.

\footnotetext{
${ }^{9}$ FRASHËRI (1964).

${ }^{10}$ IsLami (1985a). Cf. aussi IsLAmi (1985), article qui permet de mesurer l'emprise politique sur l'archéologie.

11 Lama, Dhama, Xhafa (1984), p. 59.

12 Pour le cas albanais, $c f$. CABANES \& CABANES (1999), p. 191-198, et DAvis (2000). Au sujet de la Grèce, $c f$. Hamilakis (2007), et Damaskos, Plantzos (2008), en particulier les contributions de Yannis HAMILAKIS, «Decolonizing Greek archaeology: indigenous archaeologies, modernist archaeology and the post-colonial critique», p. 273-284, et de Mark MAZOwER, "Archaeology, nationalism and the land in modern Greece», p. 33-41. En ce qui concerne la Gaule, cf. BRUHNS (2005) et REDDE (2009); et de manière plus générale et critique, lire KAESER (2000) et Bibracte (2006).

13 Citation reprise dans ARON (1983), p. 168.
} 


\section{La raison et les effets $\mathrm{du}$ « nationalisme archéologique $»^{14}$}

L'archéologie, organisée très tôt après la libération, est manifestement, pour le nouveau régime, un aspect fondamental de la recherche en sciences humaines et sociales. Le premier service archéologique albanais nait dans le cadre institutionnel de la Section d'histoire de l'Institut des Sciences en 1947. La Section d'histoire est remplacée dès 1955 par l'Institut d'histoire et de linguistique, qui comporte une section d'archéologie ${ }^{15}$. À la fin des années 1960, Enver Hoxha infléchit l'idéologie d'État vers un communisme national qui décrète, comme le montre Pierre Cabanes, l'autochthonie des Illyriens, leur origine pélasgique, et leur descendance albanaise ${ }^{16}$. En 1972, est fondée l'Académie des Sciences, héritière de l'Institut des Sciences, qui émancipe les archéologues et les rassemble en 1976 dans un Centre de recherches archéologiques, qui devient en 1991 l'Institut archéologique d'Albanie ${ }^{17}$. Les publications sont très encouragées puisque dès l'origine les archéologues sont invités à publier dans le Bulletin për Shkencat Shoqërore (Bulletin des sciences sociales), puis dans Studime Historike (Études historiques), enfin dans Iliria, revue réservée à l'archéologie, ou dans Monumentet, périodique lié à l'Institut des monuments de culture.

En 1976, année de la nouvelle constitution albanaise, les archéologues rédigèrent, sans doute collectivement, puisque le texte n'est pas signé, un programme scientifique très précis ${ }^{18}$. Un thème doit être traité aux époques préhistorique et protohistorique, antique, et médiévale : les Illyriens, leur « ethnogenèse ", leurs villes ou cités, et leur rapport historique et ethnique avec les Albanais. Il est avant tout question d'histoire et d'archéologie "politiques et sociales » des sites de l'arrière-pays. Est abordé le problème des colonies : « Nos études demeureraient incomplètes et unilatérales si elles ne s'étendaient pas avec toute l'attention requise sur les colonies grecques du littoral illyrien, en tant que partie intégrante du monde illyrien ${ }^{19}$. Sur le territoire illyrien, ces colonies

14 Cabanes \& Cabanes (1999), p. 191-198, et Cabanes (2004).

15 DuCELLIER (1967), p. 127.

16 CABANES (2004), p. 118-121.

17 CABANES (1998), p. 52.

18 Programme (1976). Comme l'écrit Pierre Cabanes en commentant un texte d'Enver Hoxha de 1969 sur la préférence vers l'étude de la genèse des Illyriens au détriment de celle des vestiges gréco-romains, "pour le dictateur albanais, une bonne archéologie est nécessairement une archéologie sélective » (CABANES [2004], p. 119).

${ }^{19}$ En réalité, les savants albanais font ici preuve d'une habileté salutaire, car insister sur le caractère illyrien des colonies grecques est manifestement un moyen de les étudier en toute quiétude idéologique. En 1998, Aleksandra Mano évoque la pensée d’Aleks Buda (MANO [1998] p. 136) : "Albanian scholars have never considered the hellenic colonies of the illyrian coast as 'enclaves' ethnically and culturally 'alien' but as part and parcel of a historical reality »- la phrase est aussi citée par Muzafer Korkuti dans le même ouvrage (p. 45). En effet, dans le texte original de 1974, A. Buda insiste ensuite sur les nombreux liens tissés entre Grecs et Illyriens (BUDA [1974], p. 55, et p. 43-44 de la publication en français dans Iliria), afin de justifier l'étude des sociétés coloniales. 
grandirent et se renforcèrent. Elles devinrent les centres économiques et culturels les plus florissants de l'Adriatique et prirent place parmi les grandes cités du monde méditerranéen. [...] L'heure est venue de nous tourner vers elles avec plus d'attention, car nous y trouverons à coup sûr la réponse à de nombreux problèmes de l'histoire illyrienne. On peut dire sans hésiter que l'étude insuffisante des matériaux recueillis dans ces cités a commencé à entraver l'étude des autres cités illyriennes. » Manifestement, les chercheurs albanais, parfaitement conscients de l'intérêt scientifique de l'étude des colonies grecques des côtes illyriennes, sont contraints de nier ou d'atténuer leur composante hellénique afin que ce programme satisfasse l'illyrocentrisme d'Enver Hoxha ${ }^{20}$.

Dans le contexte colonial, l'étude des cultes se heurte donc à la disqualification du caractère grec des fondations d'Apollonia ou d'Épidamne-Dyrrhachion. En 1998 encore, pour Aleksandra Mano, dont l'importance des travaux sur Apollonia est incontestable ${ }^{21}$, les études non albanaises sur les colonies grecques d'Illyrie méridionale sont trop souvent l'occasion de "démontrer» l'arriération des Illyriens et leur dette technique et culturelle à l'égard des Grecs et des Romains 22 . Pour elle, le processus d'«illyrianization» de ces colonies remonte au premier contact entre les populations grecques et illyriennes ${ }^{23}$.

C'est dans un passé très reculé et archaïque que l'on fonde la démonstration de la grande antiquité du peuple illyrien. Le thème de l'origine pélasgique des Albanais apparaît au XIXe siècle, dans le contexte du retrait ottoman et du

20 La définition de l'homme illyrien, objectivement peu embarrassée ni nourrie par les sources antiques, devient un espace d'élaboration de l'homme albanais. Cela est particulièrement clair dans l'extrait suivant : «Selon les auteurs antiques, les Illyriens avaient pour traits marquants la droiture et l'hospitalité. Entre autres, Démosthène, Diodore de Sicile et Nicolas de Damas, nous rapportent que les Illyriens n'avaient rien de plus cher que la liberté et qu'ils ne laissaient jamais leurs hommes blessés tomber aux mains de l'ennemi; qu'ils étaient prêts à sacrifier leur vie pour défendre leurs amis et leurs proches. Ces auteurs ont exalté en particulier leurs vertus guerrières » (POLLO et PUTO (1974), p. 11). Se rencontrent ici l'expression d'un sentiment national fort, l'exaltation des valeurs de courage, d'abnégation et de solidarité nées dans le combat contre le Balli Kombëtar et les troupes allemandes, et la construction de l'« homme nouveau » albanais. Aujourd'hui, on observe sur le web une tendance forte au nationalisme sur les sites privés abordant l'archéologie en Albanie. Un diaporama insiste par exemple sur les légendes grecques des monnaies antiques découvertes en Albanie, et on ne sait finalement si l'auteur en conçoit du dépit ou si au contraire il estime que la civilisation grecque est d'origine illyrienne, à la suite par exemple des comparaisons entre le droit ou le pré-droit à l’œuvre chez Eschyle et le kanun albanais établies par Ismail Kadaré dans Eschyle ou l'éternel perdant (texte signé en janvier 1985, et publié en français en 1988), qui, littéralement, «retrouve dans l'Orestie des éléments du coutumier albanais » (p. 105). S'il s'agit de dépit, il est un exemple de plus de la crise de l'identité nationale mise en valeur par CABANES \& CABANES (1999) à la chute du régime autoritaire. On assiste quoi qu'il en soit à une nouvelle phase dans l'histoire de l'instrumentalisation de l'Antiquité en Albanie.

${ }^{21}$ Cf. le recueil de ces travaux, MANO (2006).

22 MANO (1998), p. 137

23 MANO (1998), p. 135 : «The process of illyrianization of the hellenic colonies both in Dyrrhachion and Apollonia started with the first contact between the two populations. » 
«réveil » des nationalités balkaniques ${ }^{24}$. Bien plus tard, Enver Hoxha reprend le motif dans la même perspective nationale ${ }^{25}$. Nous sommes ici face à une position dogmatique, articulée au thème de l'autochthonie des Albanais, qui n'est pas favorable à l'étude de la vie religieuse antique. La publication par Halil Myrto d'un aguieus d'Apollon à Apollonia d'Illyrie en offre un bon exemple ${ }^{26}$, car il s'agit d'une recherche sur un objet religieux, cultuel, qui aborde le sujet sans nier théoriquement cette dimension. Néanmoins, après une étude archéologique de l'« obélisque » d'Apollon, l'aspect religieux est réduit à sa valeur symbolique, et le monument devient proprement illyrien, très ancien, et aniconique. La démonstration est ici particulièrement paradoxale, puisque le monument renvoie en réalité au culte d'un Apollon dorien patronnant la colonisation de tradition corinthienne, en particulier en Épire et en Illyrie méridionale ${ }^{27}$. Or, à cette époque, les représentations de l'aguieus à Ambracie ou l'exemplaire corcyréen datant de l'époque archaïque étaient parfaitement connus. À l'occasion de ses réflexions sur l'aniconisme, développées aussi par Moikom Zeqo dont nous aborderons les travaux, l'auteur utilise le texte d'Hérodote sur Dodone, destiné à illustrer l'ancienneté du sanctuaire épirote, et qui devient un texte théologique illyrien: les dieux des Pélasges sont anonymes - ce qui ne signifie pas qu'ils sont aniconiques -, et les Illyriens tiendraient d'eux cette caractéristique religieuse. Retenons que le kiôn d'Apollon, incontestablement lié à une région plus vaste que l'Illyrie méridionale, devient ici un monument cultuel ou culturel illyrien. À l'évidence, anonymat et aniconisme des dieux illyriens, à défaut d'être démontrables, ont un rapport étroit avec l'athéisme instauré par le régime hoxhiste.

24 DE RAPPER (2009), avec la bibliographie. De même, on peut estimer que l'usage moderne du mot « Illyrie » remonte à Napoléon Ier, $c f$. WALLACE (1998).

25 CABANES (2004), p. 119. Les doutes au sujet de la «doctrine pélasgique» exprimés par Skendër Anamali dans POLLO et PUTO (1974), p. 8, sont d'autant plus remarquables : "Quant aux Pélasges, que certains auteurs antiques citent comme les très anciens habitants des Balkans du sud et que certains érudits modernes ont considéré comme les lointains ancêtres des Albanais, aucune donnée sérieuse n'a été recueillie jusqu'à ce jour établissant un lien de succession entre eux d'une part et les Illyriens et leurs descendants, les Albanais, de l'autre. De nouvelles recherches et de solides travaux scientifiques sont nécessaires pour étayer cette hypothèse. » Au milieu des années 1990, interrogé sur le thème de l'autochthonie des Albanais, Aleks Buda, dans HaxHIMIHALI (1994), p. 56, réagit ainsi : «On peut assurément étudier ce sujet avec plus de discernement de nos jours, en écartant par exemple bon nombre de conceptions fantaisistes : la théorie pélasgienne, celle des Étrusques. Mais l'autochthonie des Albanais est un fait établi. Après de nombreuses recherches dans le domaine de l'histoire, de la linguistique, de l'archéologie, de l'ethnographie, on peut l'affirmer sans verser pour autant dans le nationalisme. »

${ }^{26}$ MYrTo (1988).

${ }^{27}$ Cf. Quantin (2011), et Quantin s. p., n. 15, avec la bibliographie. 


\section{Une Antiquité « désenchantée »}

Dans la République populaire d'Albanie, les sciences humaines doivent avant tout servir les intérêts de l'État et de ses dogmes. Les études sur la religion, quelle que soit la période considérée, ont ici un objectif idéologique qui dépasse la katharsis imposée par l'idée marxiste-léniniste - connaittre les traditions religieuses albanaises, «les véritables racines sociales des mauvaises coutumes» écrit E. Hoxha en 196928 , pour mieux les extirper de l'âme nationale -, puisqu'il s'agit aussi d'effacer les particularismes fondés sur la diversité des religions pratiquées par les Albanais (islam sunnite, islam bektashi, chrétiens orthodoxes, chrétiens catholiques). Cette fermeture engendre aussi l'asphyxie intellectuelle de la recherche albanaise : les nouvelles données de l'archéologie méditerranéenne postérieures à la Seconde Guerre mondiale, les voies méthodologiques empruntées par des chercheurs comme Jean Rudhart, Walter Burkert, les membres de l'École de Paris ou celle de Rome pénètrent difficilement en Albanie ${ }^{29}$.

Dans la préface de la synthèse historique dirigée par Stefanaq Pollo et Arben Puto, Maurice Baumont formule la vulgate hoxhiste ${ }^{30}$ : les Albanais sont « un peuple médiocrement religieux », au point que «l'activité religieuse a disparu en Albanie », car "le catholicisme était compromis par l'occupation italienne», et « l'islamisme présentait un redoutable obstacle à la libération de la femme ». Dans ce texte apologétique, nulle mention claire d'un fait qui explique à lui seul la médiocrité religieuse de l'Albanie : l'interdiction du culte promulguée par Enver Hoxha en 1967. On évoque simplement la contradiction entre la liberté des cultes et les principes du marxisme-léninisme. Il faudrait donc croire que l'interdiction de la pratique religieuse ne ferait que sceller une faiblesse nationale et confirmer une longue évolution. Mickaël Wilmart a parfaitement montré qu'il n'en était rien $^{31}$ : l'athéisme «naturel » des Albanais est une reconstruction, une « révision » de l'histoire de ces régions. À partir de 1967, l'athéisme est en réalité l'objectif d'un long processus d'enquête statistique sur les indices confessionnels et d'une « conversion » vigoureuse à un monde sans Dieu.

Dans le domaine de l'Antiquité, cette conception a joué un rôle important. En 1974, Sk. Anamali écrit que «la religion des Illyriens durant la première période du Fer n'est pas bien connue. Comme les autres peuples primitifs, ils adoraient tout ce qui leur semblait extraordinaire dans la nature, en premier lieu le soleil. [...] Les divinités anthropomorphes, aux fonctions bien définies, n’apparaitront

${ }^{28}$ Hoxha (1969), p. 160-161, cf. Wilmart (2005), p. 3.

29 Pas plus que ne furent audibles, par exemple, les voix d'Émile Durkheim, Max Weber, Marcel Mauss, Georges Dumézil, Paul Ricœur ou René Girard, si importantes dans l'historiographie du XXe siècle.

30 Pollo et Puto (1974), p. XVIII et XIX.

31 Cf. Daniel (1989), Cabanes \& Cabanes (1999), p. 173-181. Lire aussi Wilmart (2005), en particulier son commentaire des textes d'Hulusi Hako sur l'athéisme viscéral des Albanais, et de l'ouvrage d'Elira Çela, Tradita afetare tëpopullit shqiptar, Tirana, 1991. 
que plus tard $\aleph^{32}$, sous l'influence du panthéon grec. En d'autres termes, la religion gréco-romaine s'impose d'autant plus facilement que le sentiment religieux des Illyriens n'est pas vif et que leur panthéon est naturaliste et peu structuré. Comme à l'époque de la conquête ottomane, la religion est en quelque sorte perçue comme un phénomène exogène, qui, devenu indigène, n'en demeure pas moins étranger aux traditions illyriennes ou albanaises. La religion, quand elle est un système organisé, est celle de l'Autre ${ }^{33}$.

\section{Approche archéologique des sanctuaires et histoire religieuse}

Si dans le champ des sciences humaines et sociales la piété ne fut pas abordée, il serait bien entendu faux de penser que les archéologues ont nié l'existence des sanctuaires antiques ${ }^{34}$. De même, si la recherche actuelle connaît une si rapide mise à jour thématique et méthodologique, c'est grâce à cette continuité de la pratique archéologique : sous le régime hoxhiste, ce n'est certes pas la dimension religieuse des sanctuaires qui est mise en avant, mais la fouille ne peut complètement éluder les aspects cultuels quand ils sont patents.

Les précautions des chercheurs albanais, à une époque où les études sur les religions antiques, dans une perspective marxiste ou non, sont nombreuses en Europe et outre-atlantique, sont aussi perceptibles grâce au traitement scientifique réservé aux sites clairement cultuels. Après le départ de L.M. Ugolini, les principaux vestiges de Bouthrôtos composent un remarquable asclepieion, équipé d'un péribole, de temples ${ }^{35}$, d'un théâtre ${ }^{36}$, d'un enkoimeterion ${ }^{37}$, d'un thesauros-tronc ${ }^{38}$, d'un vraisemblable thesauros-oikos, et documenté par des dédicaces épigraphiques ${ }^{39}$ et des offrandes. Il s'agit d'un très beau dossier d'archéologie et d'histoire reli-

32 Pollo et Puto (1974), p. 11.

33 Le bektashisme est par exemple un magnifique contre-exemple de ce dogme historiographique. Il s'agit d'une confrérie musulmane fondée par Hadji Bektash au XIII siècle en Anatolie, et qui s'organisa véritablement au XVI ${ }^{e}$ siècle. Ce mouvement religieux s'apparente d'un point de vue doctrinaire au soufisme. Il n'a pas ralenti le détachement des Albanais vis-à-vis de la Porte, mais bien au contraire encouragé le développement du nationalisme albanais. Cf. CLAYER (1990) et (1995).

34 Citons les travaux de Skendër Anamali à Amantia et à Apollonia, où le savant découvre un sanctuaire à l'ouest de la ville, exploré récemment par une équipe américano-albanaise (terrain Bonjakët), d'Apollon Baçe, de Guri Pani à Shtyllas, ou d'Halil Myrto à Durrës.

35 Situé sur une terrasse au nord du théâtre, cet édifice a lui aussi été découvert par l'archéologue italien (UGOLINI [1937], p. 122-123, et fig. 71, p. 126). M. Melfi propose de loger ici le dieu principal du sanctuaire, Asclépios, cf. MELFI (2006).

36 Cf. GiLKES et al. (2003).

${ }^{37}$ Un portique, comme l'avait montré G. Pani, doit correspondre à un enkoimeterion, cf. MELFI (2006).

38 Cf. Ugolini (1942), p. 96-97, pl. 88, fig. 2 : dit «ara di Filisto ». La démonstration est réalisée par GORRINI, MELFI (2002), cf. ChronARG [2005], 05.08.

39 CIGIME II, n 170 à 189 bis. 
gieuses. Or, pendant la période hoxhiste, les scientifiques albanais s'intéressent à d'autres édifices, à la nécropole ${ }^{40}$, aux fortifications ${ }^{41}$, tous parfaitement dignes d'intérêt, mais désertent le cœur du site, le sanctuaire d'Asclépios, où les fouilles de L. M. Ugolini n'avaient pas épuisé la matière. Pierre Cabanes et Faik Drini furent les seuls à étudier un aspect de la vie religieuse à Bouthrôtos : les actes d'affranchissement, naturellement gravés dans le sanctuaire, sur les murs de parodos du théâtre, et mentionnant les prêtres d'Asclépios et de Zeus Sôter ${ }^{42}$. L'étude du sanctuaire antique et de la vie religieuse est ici clairement évitée.

Souvent, les archéologues ne retiennent du sanctuaire que sa dimension architecturale. Le grand temple extra-urbain de Shtyllas à Apollonia a été sondé par Camillo Praschniker, et dans la suite des travaux de Henri Daumet au XIX ${ }^{\text {e }}$ siècle, a été étudié dans les années 1980 par un architecte de l'Institut des Monuments culturels, Guri Pani, qui lui consacre une solide étude, sans qu'une fouille paraisse nécessaire ${ }^{43}$. Le temple de Lëmi i Peçit à Amantia, dans l'intérieur des terres, subit le même traitement, c'est-à-dire qu'une courte étude architecturale lui est consacrée par Apollon Baçe ${ }^{44}$, le matériel erratique est commenté ${ }^{45}$, mais le temple et surtout son environnement immédiat ne sont pas explorés systématiquement par une fouille ou des sondages. Les chercheurs s'intéressent à l'architecture du temple, mais presque jamais au sanctuaire, à l'espace sacré.

Une autre raison explique le manque d'intérêt des chercheurs pour les rites et la vie religieuse antiques: la distinction entre religion et mythologie n'est pas toujours faite clairement. En 1989, Moikom Zeqo rédige par exemple un article ${ }^{46}$, repris et traduit en français en 1995 sous la forme d'un ouvrage intitulé Aspects de la mythologie illyrienne. L'auteur estime que les Illyriens ont joué un rôle important dans l'élaboration des mythes grecs comme ceux de Kadmos et Harmonie, des Argonautes, des nostoi, et d'Héraklès, en particulier quand il évolue en Épire et en Illyrie. Aux maigres indices que les sources littéraires et très rarement épigraphiques $^{47}$ nous fournissent au sujet des divinités des Illyriens, M. Zeqo ajoute des réflexions sur l'aniconisme, la valeur religieuse des décorations géométriques, le dendromorphisme et le zoomorphisme. Mis à part le caractère suranné de ces concepts, observons que la vie religieuse illyrienne est ici conçue comme une sorte de naturalisme mâtiné d'animisme et de totémisme. Il n'y a donc pas négation de la vie religieuse, d'autant moins qu'il ne s'agit pas ici d'étudier une religion " étrangère » comme le polythéisme gréco-romain, ou les monothéismes chrétien

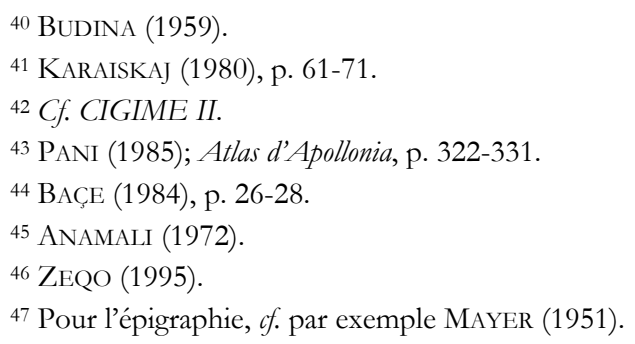


et musulman. Mais on observe une réduction de la vie religieuse à des formes élémentaires, au contenu attendu ou bien peu dense. L'auteur ne fait pratiquement pas de place à l'activité sacrificielle, et aborde très rarement le thème des rites. Une fois seulement il argumente au sujet d'un rite, réel ou supposé, interprété comme un sacrifice : «Archéologiquement, on a constaté un ancien rite qui remonte au Néolithique, qui consiste à enterrer le mort à l'intérieur du territoire du site, en position couchée, les jambes repliées et sans mobilier funéraire. Ce culte qui a survécu jusqu'à la première période du Bronze était lié au sacrifice offert aux forces qui protégeaient le site. C'est un culte illyrien exceptionnellement antique, dont le nom même est absent; il est donc aniconique. $»^{48}$ Tous les ingrédients sont ici réunis : haute antiquité vénérable, permanence, caractère illyrien, aniconisme.

Il arrive néanmoins que certaines divinités dont on découvre des représentations sculptées soient dites illyriennes, comme un dieu de l'abondance dont l'iconographie est singulière et dont la présence est bien attestée en particulier à Amantia ${ }^{49}$. Dans ce cas il est presque certain que nous avons affaire à une divinité locale. Mais le raisonnement a aussi été tenu pour Artémis ${ }^{50}$, une divinité clairement d'origine coloniale, à défaut d'être profondément corinthienne ${ }^{51}$.

Sur ce thème, l'anthropologie et l'ethnologie ont joué un rôle important, en confisquant en quelque sorte l'étude du religieux. L'œuvre de Mark Tirtja, chercheur à l'Institut de Culture populaire de l'Académie des Sciences d'Albanie, est à cet égard exemplaire. Pour lui, les faits religieux gréco-romains s'inscrivent dans une histoire longue et continue qui va de l'imaginaire illyrien des époques anciennes au folklore albanais médiéval, moderne, voire contemporain. En 1980, l'ethnologue publie une étude sur les «Croyances futiles et populaires en Albanie et leur extirpation après la libération $»^{52}$. Mais le cœur de son travail étudie les récits légendaires ${ }^{53}$, la continuité des traditions religieuses en Albanie depuis l'Antiquité illyrienne ${ }^{54}$. En forçant le trait, l'ethnologue établit le plus souvent, in fine, une perspective syncrétique : l'homme albanais est religieux, mais sa religion s'inscrit

48 ZEQO (1995), p. 10-11 (69 en fr.).

49 AnAmali (1972), p. 85-86, et 118.

50 Cf. DuCELlier (1967), p. 132, qui se fait l'écho des recherches albanaises : afin de démontrer l'existence d'établissements illyriens sur le site des futures fondations coloniales grecques, on défend l'idée d'une "présence à Apollonie même du culte illyrien d'Artémis chasseresse ». Ce n'est pas ici le fait de postuler l'existence d'un site illyrien antérieur à Apollonia - hypothèse parfaitement légitime puisqu'elle repose sur une source antique (Étienne de Byzance, s. v. 'A $\pi$ o $\lambda \lambda \omega v i \alpha$ ), mais qui pour l'heure n'a pas été démontrée par le matériel céramique découvert dans les sondages effectués dans le centre d'Apollonia, of. Stéphane Verger dans MEFRA 121-1 (2009), p. 264-268 - qui nous intéresse, mais l'instrumentalisation historiographique d'Artémis, qui, pour servir l'hypothèse d'une présence antérieure illyrienne sur le site d'Apollonia devient une déesse locale.

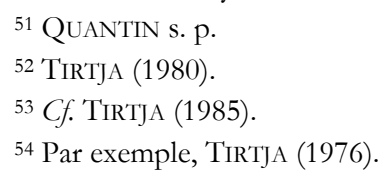


dans une continuité qui plonge ses racines dans des temps «primitifs » et dont les vestiges restent observables aujourd'hui. Néanmoins, dans le contexte hoxhiste, il demeure que l'œuvre de Mark Tirtja est remarquable car elle aborde la vie et l'imaginaire religieux ${ }^{55}$.

Du côté des spécialistes de l'Antiquité, on met volontiers en valeur le caractère primitif de la religion, un naturalisme sympathique et naif ${ }^{56}$. La vie religieuse appartient donc à une sorte de passé intemporel. Dans ce paysage, un article d'Apollon Baçe publié en 1984 est lui aussi remarquable, puisqu'il porte sur la croyance (besim) et l'architecture cultuelle antiques chez les Illyriens ${ }^{57}$. Après quelques propos généraux conformes à l'esprit du temps, l'auteur décrit les lieux de culte des colonies, puis les sanctuaires des villes illyro-épirotes, parmi lesquels figure Dodone. Cette annexion historiographique n'est pas légitime, car c'est bien la religion grecque qui fournit les clefs de compréhension du sanctuaire épirote ${ }^{58}$. Néanmoins, transgresser les frontières modernes et adopter une vue plus large tenant compte des autres recherches balkaniques est une démarche nouvelle en Albanie et louable, particulièrement dans le domaine de l'histoire et de l'archéologie cultuelles. Il est le premier, je crois, à observer que colonnes et chapiteaux octogonaux sont particulièrement bien attestés dans ces régions illyro-épirotes, de Dodone à Bouthrôtos, Phoinikè ou Apollonia. A. Baçe s'intéresse aussi aux divinités, quelque peu aux rites et utilise des concepts dont l'emploi est généralisé à cette époque, mais pas encore en Albanie.

En règle générale, les études religieuses sur l'Antiquité, qui ne devraient avoir en soi rien de subversif ${ }^{59}$, ne sont pas interdites, mais elles sont de fait négligées, car l'archéologie sous Enver Hohxa a une perspective, illyrienne, et une fonction, la fondation d'une identité nationale. Il n'est naturellement pas question que cette identité puisse reposer sur une religion, fût-elle antique, et l'on préfère insister sur une sorte d'opportunisme albanais en matière religieuse, s'exprimant dans le contexte d'une indifférence généralisée à la dimension cultuelle ou spirituelle. On ne voit guère, alors, pourquoi il aurait fallu fermer autoritairement les édifices cultuels en $1967^{60}$. Si l'étude de la religion antique en Albanie est négligée, ce n’est

55 Achevé en 1976, son ouvrage sur la mythologie des Albanais (Mitologïa ndër Shqiptarë, Tirana) ne fut publié qu'en 2004, et son auteur évita de peu la relégation grâce à l'intervention du président de l’Académie des Sciences, Aleks Buda, comme le rapporte WILMART (2005), p. 9. Les études ethnographiques et anthropologiques sont, comme l'archéologie, encouragées par le régime, qui crée dès 1948 le Centre de Recherches folkloriques.

56 On exhume un folklore "rhapsodique ", auquel un très beau roman d'Ismail Kadaré, Le dossier $H$, est consacré.

57 BAÇE (1984).

58 Cf. Mylonopoulos (2006), Dieterle (2007), et Quantin (2008).

59 Mais on sait l'usage contemporain des sources antiques qui peut être fait dans les Balkans, cf. CABANES (1988), p. 337-339.

${ }^{60}$ CABANES \& CABANES 1999, p. 173-181. On passe alors à un « athéisme d'État » (cf. WiLMART [2005], p. 2 sq). 
donc pas par négligence, mais par un oubli délibéré61. Le contraste est fort avec la Grèce de la même époque, puisqu'on observe là une valorisation de l'étude de la religion antique, car, comme la langue, elle est recognitive de la culture hellénique, aujourd'hui comme hier ${ }^{62}$. Si du côté albanais l'horizon illyrien a sclérosé la recherche sur la vie religieuse antique, du côté grec, le chêne de Dodone a fait de l'ombre à ce type d'études. Après la Seconde Guerre mondiale, c'est bien entendu Sôtiris I. Dakaris qui fut à l'origine d'un renouvellement profond de l'archéologie épirote, suivi en particulier par Chryséis Tzouvara-Souli. Les fouilles de Dodone reprennent ${ }^{63}$, et de vastes enquêtes monographiques sont menées ${ }^{64}$, que viennent compléter aujourd'hui la fouille de nouveaux sanctuaires ${ }^{65}$. La fouille du complexe de Mésopotamon par S.I. Dakaris est à cet égard symptomatique. L’archéologue, malmené par la dictature des colonels, est à la recherche du lieu de consultation des ombres des morts par Ulysse. Influencé par un érudit local, Spyros G. Mousélimi, il entreprend la fouille d'un site fortifié au sommet d'une colline du Phanari, la basse vallée de l'Achéron antique. Très vite, il propose de situer là la consultation d'Ulysse et le sanctuaire nécromantique mentionné par Hérodote en Thesprôtie, alors que le plan de l'édifice, son évidente fonction de stockage, la présence d'une grande citerne et la découverte d'éléments d'une catapulte plaident en faveur d'une interprétation très différente ${ }^{66}$. Dans le sud de l'Albanie des vestiges du même type découverts à Malathré, Çuka ou Metoq, sont, à juste titre, analysés du point de vue de l'organisation du territoire, de son exploitation et de sa défense ${ }^{67}$.

\section{Le réenchantement de l'Illyrie méridionale}

Dès 1994, Aleks Buda aborde avec lucidité ce sujet, identifie la part de doctrine contenue par cette approche, et encourage la jeune génération à combler cette lacune de la mémoire albanaise : « Dans le domaine de l'histoire contemporaine, je voudrais qu'on cesse d'envisager exclusivement les seuls aspects diplo-

61 À lire les historiens albanais, on a souvent l'impression, en particulier en conclusion des travaux, d'un exercice de style lyrique. L'approche épique, parfois lyrique de l'histoire séduit aussi les historiens « occidentaux » : Maurice Baumont, dans POLLO et PUTO (1974), cite Ronsard pour parler de l'Épire antique comme de l'épopée de Scanderberg, ou l'Andromaque de Racine (p. VIII et IX). Une des raisons « techniques » que l'on peut évoquer pour expliquer ce lyrisme est le divorce entre l'archéologie et l'histoire (cf. CABANES [2004], p. 121).

62 DAKARIS (1963).

63 DieTERLE (2007), p. 14-15.

${ }^{64}$ Par exemple, Tzouvara-Souli (1979) et (1988-1989).

65 Les sanctuaires de Dourouti en Molossie, voir Andreou, Gravani (1997) et ANDreOU (2004), ou de Mastilitsa en Thesprôtie, cf. CHristophilopoulou (2004).

${ }^{66}$ BAATZ (1982). Cf. FOUACHE, QUANTIN (1999).

${ }^{67}$ Cf. SHPuZA (2009), p. 499-501, avec les références aux fouilles et à l'analyse de ces vestiges. À Metoq, L.M. Ugolini pensa pouvoir restituer un temple. 
matiques et militaires, qu'on fasse enfin une histoire des partis politiques, de la culture et de la pensée politique. Il faudrait pour cela une préparation plus complète, plus générale des jeunes chercheurs... Et les archives doivent être ouvertes, sans limitation politique en particulier. Il y a aussi le domaine de l'histoire religieuse, ce contexte de coexistence entre trois religions en Albanie, qui ont encore été peu étudiées. Voilà de belles perspectives de recherche pour la jeune génération qui est plus libre, moins endoctrinée et moins disposée à accepter les slogans ou les solutions schématiques. ${ }^{68}$ Parallèlement à cette prise de conscience historiographique, on assiste manifestement à une fréquentation renouvelée et modérée des lieux de culte qui ont échappé à la destruction pour des raisons patrimoniales, comme l'église Sainte-Marie de Pojani, sur le site de l'antique Apollonia, où le pope Socrate restaure la liturgie orthodoxe, ou comme la mosquée Et'hem Bey de Tirana où prêche l'imam Naci ${ }^{69}$.

La vie religieuse antique est aujourd'hui un objet scientifique normal, et plutôt privilégié par la recherche, qu'elle soit menée en collaboration avec des équipes étrangères ou strictement albanaise. Parmi ces études portant sur l'archéologie cultuelle, il faut relever les travaux menés à Apollonia (Bonjakët, Shtyllas), aux temples de la région d'Épidamne-Dyrrhachion, les études sur les terres cuites du musée de Durrës, les figurines de la grotte de Konispol, la reprise de l'analyse du temple au sommet de l'acropole de Phoinikè (cf. le répertoire topo-bibliographique ci-dessous, p. 198), ou la revalorisation de l'hypothèse cultuelle au sujet des dépôts de céramique au nord de la colline 104 d'Apollonia, auparavant considérés comme les rebuts d'un atelier ${ }^{70}$. En Illyrie méridionale et en Chaonie, le réenchantement emprunte plusieurs chemins. Le mobilier découvert dans les sanctuaires fait l'objet d'études prenant en compte leur dimension votive, ce qui est nouveau en Albanie. L'équipe d'Arthur Muller à Épidamne-Dyrrhachion pratique certes une archéologie de l'artisanat, mais s'intéresse aussi aux formes de piété, à la restitution de ce matériel votif à un sanctuaire de la cité. Ce sont aussi des découvertes anciennes que Maria Grazia Amore s'est employé à étudier à Konispol, une grotte du sud de l'Albanie, dont on ignorait tout de la fonction cultuelle antique, alors que les phases les plus anciennes étaient publiées depuis fort longtemps. Du côté albanais, on aboutit à une situation paradoxale : avant comme après la colonisation corintho-corcyréenne, la vie religieuse illyrienne, locale, est mal connue, alors que les chercheurs s'intéressent maintenant à la vie religieuse gréco-romaine ${ }^{71}$.

68 A. Buda, dans Haxhiminali (1994), p. 57.

${ }^{69}$ BОTI (2009). On assiste aussi à une dissociation de l'appartenance confessionnelle et de l'identité nationale, lire DE RAPPER (2002).

${ }^{70}$ Delouis, Lamboley, Lenhardt, Quantin, Skenderaj, Verger, Vrekaj (2007), p. 43-44.

${ }^{71}$ Les études albanaises d'histoire religieuse antique sont aujourd'hui nombreuses. Citons, sans exhauxtivité, les travaux de Vasil Bereti sur Aphrodite à Amantia, d'Iris Pojani sur Artémis, Dionysos, Athéna et le sanctuaire de Bonjakët comme sur les temples de la région de Durrës, de Saïmir Shpuza sur la vie religieuse à l'époque romaine, de Maria Grazia Amore à Konispol, de 
Ce regain d'intérêt s'inscrit d'abord dans l'histoire longue des recherches archéologiques et historiques en Albanie, malgré les difficultés de la période hoxhiste. Un dossier documentaire montre parfaitement qu'avant la création d'une archéologie albanaise coordonnée dès 1948, et, a fortiori le développement actuel de vastes programmes scientifiques, toute recherche était dispersée, le plus souvent aveugle et sourde, condamnée par les appétits nationaux et la briéveté des travaux, à l'inefficacité. Une belle tête féminine en marbre conservée au Musée de Ioannina fut découverte en 1912 ou 1913 près du village de B $\alpha \gamma \gamma \varepsilon \lambda \alpha \tau^{\prime} \alpha$ $\Delta \varepsilon \lambda \beta_{i v o v}^{72}$. Or L.M. Ugolini signala en 1942, d'après des données observées entre 1928 et 1935, les "resti di un tempio a Dobra in Albania », indiquant par une carte que le toponyme est situé à l'est de Butrint, au nord du petit village de «Vagagliati » ${ }^{73}$. Ces vestiges sont certainement ceux que N.G.L. Hammond mentionna à l'est de Bouthrôtos et de la petite montagne nommée Mimiles qui culmine à $825 \mathrm{~m}$ d'altitude, au lieu-dit Dobra au nord de Vagalat, près de la Pavla. Les décrivant comme les ruines d'un temple de 6,20 × 6,60 m entouré d'un enclos, il estima qu'ils correspondaient à un sanctuaire extra-urbain de la région de Bouthrôtos ${ }^{74}$. Sans lien avec ces explorations, modestes il est vrai, le site a été sondé par l'Institut Archéologique dans les années 198075. Il paraît certain que les observations de ces archéologues, comme nous avions tenté de le montrer sans connaitre les lieux, autre incurie ${ }^{76}$, concernent le village albanais de Vagalat. La sculpture appartenait vraisemblablement à un ensemble architectural ou ornemental. I. Vokotopoulou datait cette tête de la fin du IVe siècle av. J.-C. et proposait de reconnaître Déméter ou Dioné. Le visage est en effet très proche de la belle statue de Déméter découverte à Dion et datée elle aussi de la fin du IV siècle av. J.-C. ${ }^{77}$. Dans le cadre des travaux de la mission italienne et albanaise de Phoinikè, Luca Mercuri a composé un catalogue raisonné et commenté des sculptures découvertes dans la capitale chaone et aux alentours. De Vagalat, proviennent un torse d'Artémis à chiton et himation, un torse d'une Déméter assise, et, comme l'a bien vu l'auteur, une tête féminine, qu'il propose aussi d'attribuer à Déméter, conservée au Musée de Ioannina, ici datée du III siècle av.

Belisa Muka sur les figurines en terre cuite, de Bashkim Vrekaj sur le nymphaion d'Apollonia, ou de Faik Drini sur l'épigraphie religieuse.

72 Vokotopoulou (1973), pl. 37. Cette découverte a certainement eu lieu après la déclaration d'indépendance de l'Albanie par Ismail Qemal à Vlora le 28 novembre 1912, l'année même qui voit l'intégration de l'Épire à l'État grec.

${ }^{73}$ Ugolini (1942), p. 21, et fig. 9, p. 11 pour la carte.

74 Hammond (1967), p. 96-97.

${ }^{75}$ Cf. les chroniques de fouille de Dh. Çondi, «Dobër (Sarandë) », Iliria 14-2 (1984), p. 266267, et 16-2 (1986), p. 262-263.

${ }^{76}$ QUANTIN (1997), p. 446-447.

77 Cf. GinouvÈs (1993), p. 107. Les deux têtes étaient décorées d’éléments métalliques, diadème et bijoux. 
J.-C. ${ }^{78}$. Or dans l'étude du paysage religieux de l'Épire antique, ce sanctuaire, dont l'histoire archéologique est ancienne, cahoteuse et incertaine, prend et prendra à l'avenir une place importante dans la réflexion sur les sanctuaires et la vie religieuse antiques dans ces régions ${ }^{79}$. En effet, les sanctuaires ruraux, dans ces régions où la cité ne s'est pas imposée comme le modèle politique et social principal, sont fort mal connus. Il conviendra alors par exemple de comparer le site de Dobër avec celui de Mastilitsa, connu depuis peu en Thesprôtie80. D'autre part, si l'attribution à Déméter se confirme, le sanctuaire de Dourouti en Molossie bénéficiera enfin d'un bon élément de comparaison, puisque jusqu'à sa découverte aucun thesmophorion ou sanctuaire de Déméter n'était connu en Épire.

L'étude de la vie religieuse antique, qu'elle soit gréco-romaine ou illyrienne, fut en Albanie victime d'un moment historique, celui de la radicalisation idéologique du régime hoxhiste à la charnière des décennies 1960 et 1970 : le dogme de l'autochthonie, la continuité décrétée entre les Pélasges et les Albanais et l'interdiction des pratiques cultuelles, ne laissaient aucune place à la religion. Dans des régions comme l'Épire et l'Illyrie méridionale, une initiative comme la chronique de Kernos, au-delà de la mise à disposition d'une documentation disparate, peut suggérer l'intérêt d'une articulation générale des travaux sur les sanctuaires antiques. Le contexte est favorable, car on assiste à une redéfinition radicale des rapports entre les intérêts scientifiques et patrimoniaux, dans le cadre d'une recherche internationale coordonnée, dont l'Albanie est en quelque sorte un laboratoire dans les Balkans.

Université de Pau et des pays de l'Adour

François QUANTIN

Département d'Histoire, Histoire de l'art et Archéologie

IRAA du CNRS (USR 3155), IRSAM,

avenue du Doyen Poplawski, B.P. 1160

F - 64013 PAU

Courriel:francois.quantin@univ-pau.fr

${ }^{78}$ Cf. Mercuri (2005a) et (2005b). L'archéologue estime, à juste titre, que des fragments de Dobër pourraient appartenir aux statues de culte d'un sanctuaire extra-urbain dédié à une divinité féminine, peut-être Déméter et Coré associées à Artémis.

${ }^{79}$ Pour les premières conclusions, $c f$. DE MARIA, MerCuri (2007), p. 150-159.

${ }^{80}$ Cf. supra n. 65. 


\section{Répertoire topo-bibliographique succinct des sanctuaires pré- romains dans le sud de l'Albanie}

Sont ici mentionnés les lieux de culte attestés avec certitude par l'archéologie. Les sanctuaires explicitement signalés par une source littéraire ou épigraphique sont retenus, à condition que cette source contienne une attribution à une communauté ou une ville précise, même si la localisation nous échappe. Les dédicaces épigraphiques ne sont pas ici prises en compte en soi. À Byllis par exemple, les dédicaces font connaitre plusieurs divinités, mais on ignore où sont les sanctuaires et leur nombre. Les lieux de provenance des dédicaces à Poséidon ne sont pas non plus signalés, puisqu'ils ne correspondent assurément pas tous à des sanctuaires antiques. Quand l'identification de la divinité est certaine, nous renvoyons simplement à la référence bibliographique fondant cette certitude. L'identité de la divinité reste dans la plupart des cas une hypothèse, proposée entre crochets, complétée par la référence de la publication qui discute cette attribution.

\section{Épidamnos-Dytrhachion}

- Artemision péri-urbain de Dautë, of. MUlLer, TARTARi (2009) et (2011).

- Temple extra-urbain de Spitalla, of. POJANI (2011), p. 338-341.

- Temple extra-urbain du cap Palla, cf. POJANI (2011), p. 341-342.

\section{Apollonia}

- Hieron d'Apollon, cf. CIGIME I-2, n 315, 1. 51.

- Temple urbain de la «colline $104 »$ [Artémis? cf. Atlas d'Apollonia, p. 249; Apollon ? cf. CEKA (2008), p. 39].

- Temple péri-urbain de Bonjakët, cf. Davis, Stocker, Pojani, Dimo, Lynch, GERKE (2011).

- Temple extra-urbain de Shtyllas [Artémis ? cf. Atlas d'Apollonia, p. 331].

- Sanctuaire oraculaire du Nymphaion aux confins du territoire, chez Dion Cassius, Histoire romaine XLI, 45 ; cf. VREKAJ (2011).

\section{Amantia}

- Temple péri-urbain de Lëmi i Peçit, cf. AnAmali (1972), p. 80-81 [Aphrodite Pandamos ? of. BERETI (2011)].

\section{Treport}

- Sanctuaire d'Athéna, connu par une estampille sur tuile marquée A@ANAC. Cf. BERETI (1977-1978), p. 286 (SEG XXXII [1982], 621) dessin dans la thèse du même auteur Vëndbanimi në Treport, p. 155 et 158, pl. LXX, 368 (cf. maintenant REA 113 [2011], p. 41-42).

\section{Grammata}

- Sanctuaire des Dioscures dans une crique, of. HAJDARI, REBOtON, SHPUZA, CABANES (2007). 


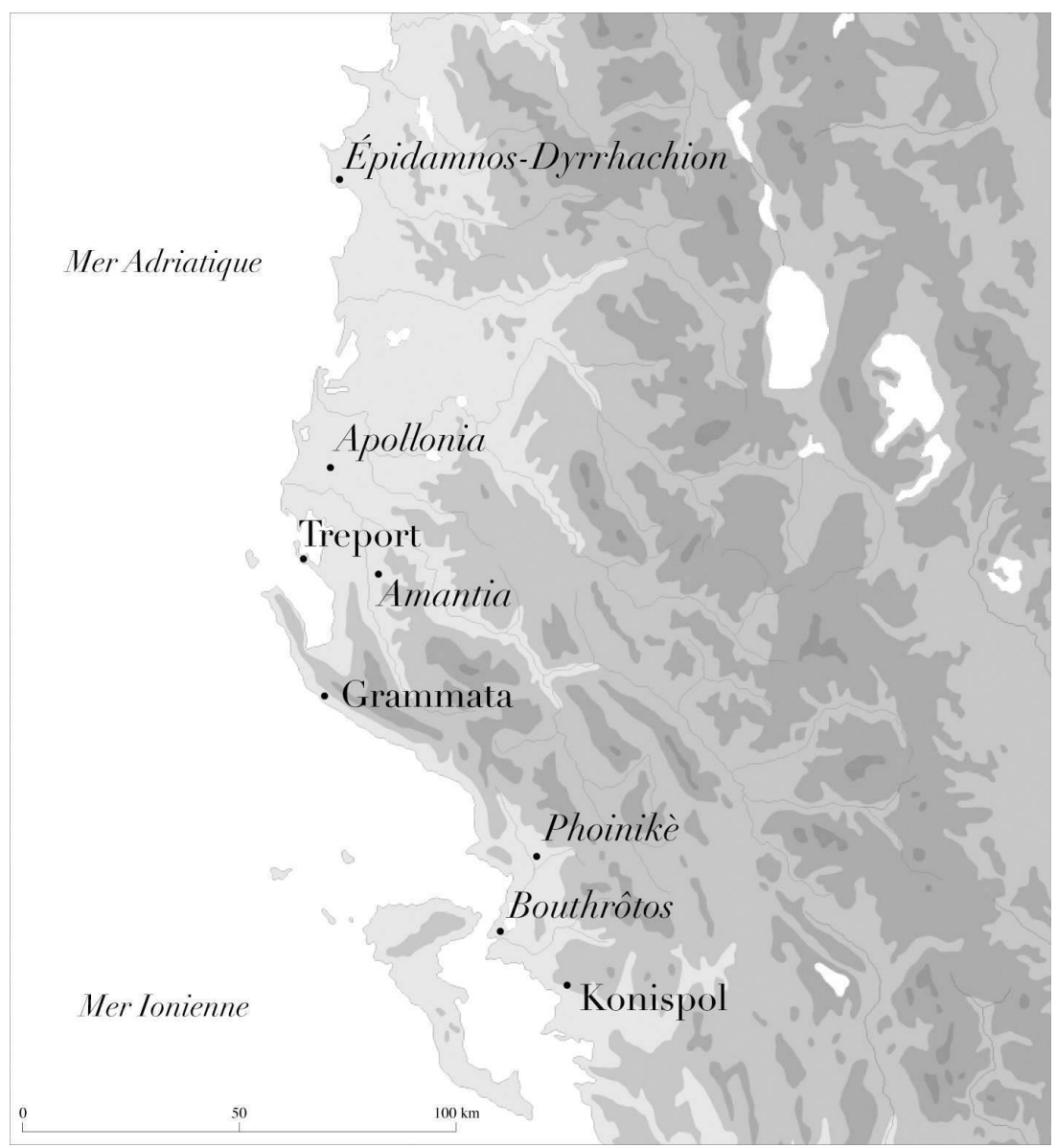

\section{Phoinikè}

- Temple urbain distyle in antis, cf. DE MARIA (2002) [Athéna Polias? cf. QUANTIN (2007)].

- Sanctuaire extra-urbain de Dobër [Déméter et/ou Artémis ?, cf. DE MARIA, MERCURI (2007), p. 156-158].

- Edificio sacro dans la ville basse, cf. LEPORE (2007), p. $92-98$ [Poséidon ? Artémis ? cf. DE MARIA, MERCURI (2007), p. 168].

\section{Bouthrôtos}

- Sanctuaire d'Asclépios, $c f$. MELFI (2006).

- Sanctuaire extra-urbain et côtier de Poséidon, chez Strabon, VII, 7, 5 [cf. QUANTIN (2004), n. 56 ; son ancienneté pré-romaine n'est pas assurée].

\section{Konispol}

- Grotte avec dépôt votif constitué de figurines en terre cuite, cf. AMORE (2006). 


\section{Bibliographie}

Amore (2006): Maria Grazia Amore, "Use and reuse of Konispol cave: The terracotta figurines", in L. BejKo, R. Hodges (éds), New Directions in Albanian Archaeology, Studies presented to Muzafer Korkuti, Tirana (International Centre for Albanian Archaeology Monograph Series, 1), p. 107-117.

AnAmali (1972) : Skënder Anamali, «Amantia », Iliria 2, p. 61-133.

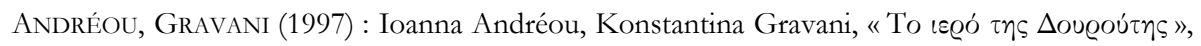
$\Delta \omega \delta \omega \omega \eta 26-1$ (1997) [1999], p. 581-626.

ANDrÉou (2004) : Ioanna Andréou, «Le sanctuaire de Dourouti: le culte et les pratiques cultuelles dans le cadre matériel », in P. Cabanes, J.-L. Lamboley (éds), L'Illyrie méridionale et l'Épire pendant l'Antiquité - IV. Actes du quatrième colloque international de Grenoble (10-12 octobre 2002), Grenoble, p. 569-581.

ARON (1983) : Raymond Aron, Mémoires, Paris.

Atlas d'Apollonia: Apollonia d'Illyrie. 1. Atlas archéologique et historique, études réunies par V. Dimo, $\mathrm{Ph}$. Lenhardt et F. Quantin, Institut archéologique d'Albanie - École française d'Athènes Ministère des Affaires étrangères - École française de Rome, Rome (Collection de l'ÉfR, 391).

BAATz (1982) : Dieter Baatz, « Hellenistische Katapulte aus Ephyra (Epirus) », MDAI 97, p. 211 233.

BAÇE (1984) : Apollon Baçe, «Vështrim mbi besimin dhe arkitekturën e kultit tek Ilirët (= Aperçu sur la foi et l'architecture du culte chez les Illyriens) », Monumentet fasc. 2, p. 5-32.

Bereti (2011) : Vasil Bereti, «Aphrodite à Amantia», in P. Cabanes, J.-L. Lamboley (éds), L'Illyrie méridionale et l'Épire pendant l'Antiquité - IV. Actes du quatrième colloque international de Grenoble (10-12 octobre 2002), Grenoble, p. 589-594.

Bibracte (2006) : L'archéologie, instrument du politique? Archéologie, histoire des mentalités et construction européenne, Actes du colloque de Luxembourg (16-18 novembre 2005), Dijon/Bibracte (Centre archéologique européen).

Bоті (2009) : Arqile Boti, «La religion, dernière illusion de la jeunesse », Courrier international 986 (24-30/09/2009), p. 22.

BRUHNS (2005) : Hinnerk Bruhns, «Grecs, Romains et Germains au XIXe siècle : quelle Antiquité pour l'État national allemand?", Anabases 1, p. 17-43.

BuDA (1974) : Aleks Buda, «Ilirët e Jugut si problem i historiografisë (= Les Illyriens du sud, un problème de l'historiographie) », Kuvendi I i Studimeve Ilire, Tirana, 1974, p. 43-64; Iliria 4 (1976), p. 39-53 (une première version avait été publiée dans la revue Studime Historike 3 [1972], p. 729).

BudinA (1959) : Dhimosten Budina, «La nécropole de Butrint », Buletin i Universitetit Shetëtor të Tiranës, Seria Shkencat Shoqërore 1959-2, p. 246-256.

Cabanes (1978): Pierre Cabanes, «Les recherches archéologiques en Albanie dans les trente dernières années », DHA3, p. 319-345.

Cabanes (1986) : Pierre Cabanes, «Recherches archéologiques en Albanie 1945-1985 », RA, p. $107-142$.

Cabanes (1988) : Pierre Cabanes, Les Illyriens, de Bardylis à Genthios (IVìme - II'me siècles avant J.-C.), Paris.

CABAnes (1996) : Pierre Cabanes, «L’École française d'Athènes en Épire et en Albanie », BCH 120, p. 397-403.

CABANES (1998) : Pierre Cabanes, «Regards sur cinquante ans d'archéologie albanaise », in 50 vjet arkeologii shqiptare, Simpozium ndërkombëtar, Tirana, 9-10 nëntor 1998, Iliria 28 (1998) [2000], p. 4972.

Cabanes \& Cabanes (1999): Pierre Cabanes, Bruno Cabanes, Passions albanaises. De Berisha an Kosovo, Paris. 
CABANes (2004) : Pierre Cabanes, «Archéologie et identité nationale en Albanie au Xxème siècle », DHA 30-1, p. 115-122.

CEKA (2008) : Neritan Ceka, Apollonia. Histoire et monuments, Tirana.

Christophilopoulou (2004) : Anastasia Christophilopoulou, «Enquête sur la topographie de la zone littorale nord de la Thesprôtie ", in P. Cabanes, J.-L. Lamboley (éds), L'Illyrie méridionale et l'Épire pendant l'Antiquité - IV. Actes du quatrième colloque international de Grenoble (10-12 octobre 2002), Grenoble, p. 191-196.

CIGIME I-2 : Pierre Cabanes (directeur), Corpus des inscriptions grecques d'Illyrie méridionale et d'Épire II, P. Cabanes, N. Ceka, Inscriptions d'Épidamne-Dyrrbachion et d'Apollonia 2, École française d'Athènes (Collection Études épigraphiques, 2).

CIGIME II : Pierre Cabanes (directeur), Corpus des inscriptions grecques d'Illyrie méridionale et d'Épire II, P. Cabanes et F. Drini, Inscriptions de Bouthrôtos, École française d'Athènes (Collection Études épigraphiques, 2).

Clayer (1990): Nathalie Clayer, L'Albanie, pays des derviches. Les ordres mystiques musulmans en Albanie à l'époque post-ottomane (1912-1967), Berlin/Wiesbaden (Osteuropa-Institut an der Freien Universität Berlin, Balkanologische Veröffentlichungen, 17).

Clayer (1995) : Nathalie Clayer, «Bektachisme et nationalisme albanais », in Alexandre Popovic et Gilles Veinstein (éds), Bektachiyya. Études sur l'ordre mystique des Bektachis et les groupes relevant de Hadji Bektach, Istanbul, p. 277-308.

DAKARIS (1963) : Sôtiris I. Dakaris, «Das Taubenorakel von Dodona und das Totenorakel bei Ephyra », Antike Kunst, p. 35-55.

Damaskos, Plantzos (2008): Dimitri Damaskos \& Dimitri Plantzos, A Singular Antiquity. Archaeology and Hellenic Identity in twentieth-century Greece, Musée Benaki, 3e supplément, Athènes.

Daniel (1989) : Odile Daniel, « Nationalité et religion en Albanie », L'Autre Europe 21-22, p. 268277.

Davis (2000): Jack L. Davis, «Warriors for the Fatherland: National Consciousness and Archaeology in Barbarian Epirus and Verdant Ionia, 1912-22», Journal of Mediterranean Archaeology 13-1, p. 76-98.

Davis, Stocker, Pojani, Dimo, Lynch, Gerke (2011) : Jack L. Davis, Sharon R. Stocker, Iris Pojani, Kathleen M. Lynch, Tammie Gerke, «Archaic Apollonia: New Light from the Bonjakët Site ", in J.-L. Lamboley, M.P. Castiglioni (éds), L'Illyrie méridionale et l'Épire dans l'Antiquité - V. Actes du Ve colloque international de Grenoble (8-11 octobre 2008), Grenoble, p. 209-214.

Delouis, Lamboley, Lenhardt, Quantin, Skenderaj, Verger, Vrekaj (2007) : Olivier Delouis, Jean-Luc Lamboley, Philippe Lenhardt, François Quantin, Altin Skenderaj, Stéphane Verger, Bashkim Vrekaj, «La ville haute d'Apollonia d'Illyrie : étapes d'une recherche en cours », in D. Berranger-Auserve (éd.), Épire, Illyrie, Macédoine ..., Mélanges offerts au Professeur Pierre Cabanes, Clermont-Ferrand (Erga, Recherches sur l'Antiquité, 10), p. 37-53.

De MARIA (2002) : Sandro de Maria, «Il Thesauròs: una revisione», in S. De Maria, Sh. Gjongecaj (éds), Phoinike I. Rapporto preliminare sulla campagna di scavi e ricerche 2000, Bologna/Firenze, p. 55-61.

De Maria, Gjongecaj (éds) (2005) : Sandro de Maria, Shpresa Gjongecaj (éds), Phoinike III. Rapporto preliminare sulla campagna di scavi e ricerche 2002-2003, Bologna.

De Maria, Mercuri (2007) : Sandro de Maria, Luca Mercuri, «Testimonianze e riflessioni sul culto di Artemide a Phoinike», in D. Berranger-Auserve (éd.), Épire, Illyrie, Macédoine ..., Mélanges offerts au Professeur Pierre Cabanes, Clermont-Ferrand (Erga, Recherches sur l'Antiquité, 10), p. $147-174$.

DE RAPPER (2002) : Gilles de Rapper, «Espace et religion : chrétiens et musulmans en Albanie du Sud », Études balkaniques 9, p. 17-39 (http:// etudesbalkaniques.revues.org/index127.html).

DE RAPper (2009) : Gilles de Rapper, "Pelasgic Encounters in the Greek-Albanian Borderland. Border Dynamics and Reversion to Ancient Past in Southern Albania », Anthropological Journal of European Cultures 18-1, p. 50-68. 
DiETERLE (2007) : Martina Dieterle, Dodona. Religionschicbtliche und historische Untersuchungen zur Entstebung und Entwicklung des Zeus-Heiligtums, Hildesheim et al. (Spudasmata, 116).

DrINI (2011) : Faïk Drini, «Divinités et cultes dans les contrées antiques de l'actuelle Albanie à travers les inscriptions", in P. Cabanes et J.-L. Lamboley (éds), L'Illyrie méridionale et l'Épire pendant l'Antiquité $-I V$. Actes du quatrième colloque international de Grenoble (10-12 octobre 2002), Grenoble, p. 583-587.

DuCELLIER (1967) : Alain Ducellier, «Les études historiques en République populaire d'Albanie (1945-1966)», RH 237, p. 125-144.

FouAChe, QuANTin (1999): Éric Fouache, François Quantin, «Représentations et réalité géographique de l'entrée des enfers de Thesprôtie », in Ch. Cusset (éd.), La nature et ses représentations dans l'Antiquité, Actes du colloque des 24 et 25 octobre 1996, E.N.S. Fontenay-St Cloud, Paris, p. 29-61.

FRASHËRI (1964) : Kristo Frashëri, Histoire d'Albanie, Tirana.

GILKES et al. (2003) : Oliver J. Gilkes (éd.), Anna Maria Liberati, Lida Miraj, Iris Pojani, Frank Sear, John Wilkes et Barbara Polci, The Theater at Butrint. Luigi Maria Ugolini's Excavations at Butrint 1928-1932 (Albania Antica IV), British School at Athens, Supplementary Volume n 35.

GinOuvÈs (1993) : René Ginouvès, La Macédoine de Philippe II à la conquête romaine, Paris.

Gorrini, Melfi (2002): Maria Elena Gorrini et Melina Melfi, «Archéologie des cultes guérisseurs : quelques observations », Kernos 15, p. 247-265.

Hajdari, Reboton, Shpuza, Cabanes (2007) : Arben Hajdari, Johany Reboton, Saimir Shpuza, Pierre Cabanes, «Les inscriptions de Grammata (Albanie) », REG 120-2, p. 353-394.

Hamilakis (2007) : Yannis Hamilakis, The Nation and its Ruins: Antiquity, Archaeology, and National Imagination in Greece, Oxford.

Hammond (1967): Nicholas Geoffrey Lemprière Hammond, Epirus. The geography, the ancient remains, the history and the topography of Epirus and adjacent areas, Oxford.

Haxhiminali (1994): Marin Haxhimihali, «Aux origines de la science historique albanaise. Entretien avec Monsieur Aleks Buda, Président honoraire de l'Académie des sciences d'Albanie », European Review of History - Revue européenne d'bistoire I-1, p. 55-57.

Heuzey (1862): Léon Heuzey, Catalogue de la Mission de Macédoine et de Thessalie, dirigée par L. Heuzey, avec la coopération de M. Daumet, architecte, Paris, Michel Lévy Frères.

Heuzey, Daumet (1876) : Léon Heuzey et Henri Daumet, Mission archéologique de Macédoine, Paris. Hoxнa (1969) : Enver Hoxha, Discours 1967-1968, Tirana.

IsLAmi (1985) : Selim Islami, « Enver Hoxha, frymëzues dhe përkrahës i arkeologjisë shqiptare (= Enver Hoxha, inspirateur et protecteur de l'archéologie albanaise) », Iliria 15-1, p. 5-14.

IsLAmi (1985a) : Selim Islami (éd.), Skënder Anamali, Muzafer Korkuti, Frano Prendi, Les Illyriens. Aperçu bistorique, Tiranë.

KAESER (2000) : Marc-Antoine Kaeser, «Nationalisme et archéologie : quelle histoire ?», Revue d'Histoire des Sciences Humaines 2000/1 ( $\mathrm{n}^{\circ}$ 2), p. 155-162.

KARAISKAJ (1980) : Gjerak Karaiskaj, 5000 vjet fortifikime në Shqipëri, Tiranë, 1980 [1981].

Lama, Dhama, Xhafa (1984) : Faik Lama, Todi Dhama, Bajram Xhafa, Historia, për klasen V të shkoollës 8-vjeçare, Shtëpia botuese e librit shkollor, Tiranë.

LEPORE (2007): Giuseppe Lepore, «La necropoli e le aeree limitrofe», in S. De Maria, Sh. Gjongecaj (éds), Phoinike IV. Rapporto preliminare sulla campagna di scavi e ricerche 2004-2006, Bologna, p. 89-119.

MANO (1998) : Aleksandra Mano, «Evoluimi i mendimit arkeologijk shqiptar për kolonizim helen në Ilirinë e Jugut (= The Evolution of the Albanian Archaeological thinking about the Hellenic Colonization of Southern Illyria)», in 50 vjet arkeologji shqiptare, Simpozium ndërkombëtar, Tirana, 9-10 nëntor 1998, Iliria 28 (1998) [2000], p. 129-137.

Mano (2006) : Aleksandra Mano, Apolonia e Ilirisë. Kërkime dhe studime arkeologjike, Tirana. 
MAYer (1951) : Anton Mayer, «Die illyrischen Götter (Vidasus und Thana)», Glotta 31, p. 235243.

Melfi (2006) : Milena Melfi, «The Sanctuary of Asclepius », in I.L. Hansen, R. Hodges (éds), Roman Butrint: An Assessment, Oxford, p. 17-32.

Mercuri (2005a): Luca Mercuri, «Sculture e scultori a Phoinikè tra età ellenistica ed epoca romana », Ocnus 13, p. 229-250.

Mercuri (2005b) : Luca Mercuri, « Nuove sculture di Phoinikè », in De Maria, GjongeCAj (éds) (2005), p. 187-194.

Muller, Tartari (2009): Arthur Muller, Fatos Tartari (éds), Artémis à Dyrrbachion, Institut Archéologique d'Albanie - École française d'Athènes - Halma/Ipel UMR 8164, Tirana (Guides de Durrës, 1).

Muller, TARTARi (2011) : Arthur Muller, Fatos Tartari, «Des figurines aux collines. Contribution à la topographie d'Épidamne-Dyrrhachion", in J.-L. Lamboley, M.P. Castiglioni (éds), L'Illyrie méridionale et l'Épire dans l'Antiquité - $V$. Actes du Ve colloque international de Grenoble (8-11 octobre 2008), Grenoble, p. 289-298.

Mylonopoulos (2006): Joannis Mylonopoulos, «Das Heiligtum des Zeus in Dodona. Zwischen Orakel und venatio ", in J. Mylonopoulos, H. Roeder (éds), Archäologie und Ritual. Auf der Suche nach des rituellen Handlung in den antiken Kulturen Ägyptens und Griechenlands, Wien, p. 185214.

Myrto (1988) : Halil Myrto, « Konsiderata mbi obeliskun e Apolonisë », Monumentet 35, p. 81-86.

PANI (1985) : Guri Pani, « Le temple à Shtyllas », Monumentet 29, p. 79-85.

PAтsCh (1926): Karl Patsch, «Aus dem albanischen National-Museum», JÖAI 23, suppl. col. 210-230.

PojAni (2011): Iris Pojani, «Le projet de la chôra antique de Durrës (Albanie). Terres environnantes d'Épidamnos-Dyrrhachion », in J.-L. Lamboley, M.P. Castiglioni (éds), L'Illyrie méridionale et l'Épire dans l'Antiquité - V. Actes du Ve colloque international de Grenoble (8-11 octobre 2008), Grenoble, p. 337-343.

Pollo et Puto (1974) : Stefanaq Pollo, Arben Puto (direct.) avec la collaboration de Kristo Frashëri et Skënder Anamali, Histoire de l'Albanie des origines à nos jours, préface de Maurice Baumont, Roanne/Lyon.

Programme (1976) : «Arkeologia shqiptare dhe detyrat e saj aktuale (= L'archéologie albanaise et ses tâches essentielles) », Iliria 6, p. 13-20.

QUANTIN (1997) : François Quantin, La vie religieuse en Épire antique, thèse de l'Université Lyon 2, à paraître.

QuANTIN (2004): François Quantin, «Poséidon en Chaonie et en Illyrie méridionale», in G. Labarre (éd.), Les cultes locaux dans les mondes grec et romain, Actes du colloque de Lyon 2, UMR 5189 du CNRS (7-8 juin 2001), Lyon/Paris, p. 153-178.

QuAntin (2007) : Séverine et François Quantin, «Le déplacement du temple d’Athéna Polias en Chaonie. Remarques sur les cosiddetti 'temples voyageurs'», in D. Berranger-Auserve (éd.), Épire, Illyrie, Macédoine ..., Mélanges offerts au Professeur Pierre Cabanes, Clermont-Ferrand (Erga, Recherches sur l'Antiquité, 10), p. 175-196.

QuANTIN (2008) : François Quantin, «Recherches sur l'histoire et l'archéologie du sanctuaire de Dodone. Les oikoi, Zeus Naios et les Naia», Kernos 21, p. 9-48.

QuAntin (2011): François Quantin, «L'aguieus d'Apollon à Apollonia d'Illyrie», in J.-L. Lamboley et M.P. Castiglioni (éds), L'Illyrie méridionale et l'Épire dans l'Antiquité - V. Actes du Ve colloque international de Grenoble (8-11 octobre 2008), Grenoble, p. 215-231.

QUANTIN s. p. : François Quantin, «Contribution à l'histoire religieuse des colonies corinthiennes occidentales. Le problème du transfert des cultes métropolitains vers les cités coloniales », Actes du colloque international de Cosenza (5-7 mai 2010), PRIN «La 'terza' Grecia e l'Occident ", sous presse.

REDDE (2009) : Michel Reddé, «Alésia et la mémoire nationale française », Anabases 9, p. 11-22. 
REY (1939) : Léon Rey, «Fouilles de la Mission française à Apollonie d'Illyrie : I, l'odéon; II, le sanctuaire; III, le musée archéologique de Valona », Albania VI, p. 5-15, pl. I-XXVI.

SHPUZA (2009) : Saimir Shpuza, "Cités et territoire. L'Illyrie méridionale à l'époque romaine», MEFRA 121, p. 481-502.

SHPUZA (2010) : Saimir Shpuza, «La religion en Illyrie méridionale pendant l'époque romaine », Studia Albanica, p. 27-41.

TiRTjA (1976) : Mark Tirtja, "Elemente të kulteve ilire te shqiptarët (= Éléments des cultes illyriens chez les Albanais - Le culte du soleil)», Iliria V-2, Premier colloque des études illyriennes, p. 241-247.

TiRTJA (1980) : Mark Tirtja, «Croyances futiles et populaires en Albanie et leur extirpation après la libération », Ethnographie albanaise 11, p. 183-215.

TirTjA (1985) : Mark Tirtja, «La mythologie dans l'épopée légendaire», L'Ethnographie 85-2, p. 33-42.

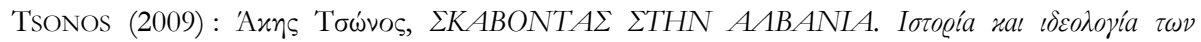

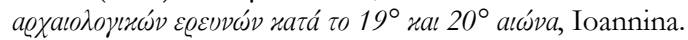

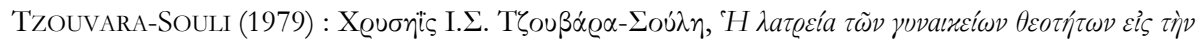
ảexaíav 'H

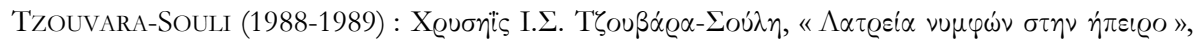
HПЕIPSTIKA XPONIKA 29, p. 9-65.

Ugolini (1937) : Luigi Maria Ugolini, Butrinto. Il mito d'Enea. Gli scavi, Rome.

Ugolini (1942) : Luigi Maria Ugolini, L'acropoli di Butrinto, collection Albania antica III, Rome.

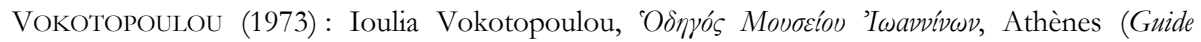
archéologique, 24).

VReKAJ (2011) : Bashkim Vrekaj, «Le nymphée d'Apollonia d'Illyrie », in J.-L. Lamboley et M.P. Castiglioni (éds), L'Illyrie méridionale et l'Épire dans l'Antiquité $-V$. Actes du Ve colloque international de Grenoble (8-11 octobre 2008), Grenoble, p. 199-207.

WAgSTAFF (2001) : J.M. Wagstaff, "Colonel Leake. Traveller and scholar», in S. Searight et M. Wagstaff (éds), Travellers in the Levant. Voyagers and visionaries, Durham, p. 3-15.

WALLACE (1998) : Jennifer Wallace, «A (Hi)story of Illyria », G \& R 45, p. 213-225.

Wilmart (2005) : Mickaël Wilmart, «Sciences humaines et athéisme d'État. Étudier le fait religieux dans l'Albanie communiste", in Sciences bumaines et religion(s), XVI $T^{e}$-XXe siècles, colloque de l'EHESS (Paris, 21-23 septembre 2005), à paraitre; disponible en ligne à l'adresse http://halshs.archives-ouvertes.fr/halshs-00120313

ZeQo (1995) : Moikom Zeqo, Aspekte të mitologiisë Ilire - Aspects de la mythologie illyrienne, Tirana (traduction française d'un article paru dans Kultura Popullore, 1990-1, p. 125-151). 